\title{
Theoretical investigation of epitaxial deformation and the hcp-bcc transition of alkali metals
}

\author{
Weixue $\mathrm{Li}^{*}$ and Tzuchiang Wang \\ Laboratory for Nonlinear Mechanics of Continuous Media (LNM), Institute of Mechanics, Chinese Academy of Sciences, \\ Beijing 100080, People's Republic of China \\ (Received 20 November 1998; revised manuscript received 9 June 1999)
}

\begin{abstract}
The deformation of alkali metals $\mathrm{K}, \mathrm{Rb}$, and $\mathrm{Cs}$ under epitaxial deformation is studied via the ab initio pseudopotential plane wave method using the local-density approximation. Under loading from the stable fcc phase, metastable states along directions [001], [111], and [201] are identified. One metastable state, presented at direction [201], has a very low symmetry in contrast to the planes [001] and [201]. Our results show that the softening direction and sequences of growth is significantly affected by the existence of the metastable states and magnitude of the energy barrier. The resulting softening sequences from soft to hard are [201], [110], [001], and [111] under biaxial compression and [001], [111], [201], and [110] under biaxial tension. An orthorhombic deformation path is used to investigate the fact, that the structure of the alkali films $\mathrm{K}$ and Cs evolve from the quasihexagonal structure into the (110)-oriented bcc structure, observed by experiments. [S0163-1829(99)06741-7]
\end{abstract}

\section{INTRODUCTION}

Recently, epitaxial growth of metal films and superlattices have attracted much interest ${ }^{1-6}$ due to their unusual physical properties. Most metal-metal superlattices have been grown for elements in different crystal structures (e.g., fcc/bcc) and with considerable size mismatch. Furthermore, elemental metals and alloys have been found to form epitaxially in structures that are unstable in the bulk form. ${ }^{7-10}$ These systems are usually strained due to film-substrate lattice mismatch. Among the various modes of epitaxial growth, pseudomorphic epitaxy, i.e., coherent epitaxy, is a special one. ${ }^{6,11-13}$ For the lattice mismatch and coherence between substrate and film, both isotropic expansive and compressive strain can be loaded on the film, while the stress perpendicular to the film vanishes. The strain is elastic and can be highly nonlinear. A stable structure of elemental metals, which are unstable in bulk form, can be found. If the structure corresponds to a local minimum of energy and is free of stress with respect to the epitaxial deformations, it is called a "phase", of the material. Such a phase will be stable or metastable depending on whether it has the lowest energy compared to other local minima. ${ }^{13}$ Metals can have various phases with different symmetry, e.g., $\mathrm{Na}$ and $\mathrm{Rb}$ (Refs. 14,15) with bcc and fcc phase, Ti and V (Refs. 15,16) with cubic and tetragonal phase. They can also have phases with other symmetries, e.g., heavy alkali metals with an hcp phase and a special phase without any simple structure, which will be shown in the following.

The objectives of the present paper are threefold: first, to investigate the isotropic epitaxial strain on the four planes [i.e., (100), (110), (111), and (201) planes], and studied possible metastable states. Second to discuss the relation between softness of epitaxial growth, the existence of the metastable state, and the magnitude of the energy barrier along the various directions in details. Finally, try to explain the structural transition of alkali metals observed by experiments. The main results are the following: (1) The metastable state of alkali metals under epitaxial deformation along directions [001], [110], and [201] are identified; one structure, presented for the case of [201], is identified. (2) With the above results, we illustrate the existence of the metastable states and the magnitudes of energy barrier determine the softening direction and sequences of epitaxial growth. (3) An orthorhombic deformation path is used to investigate the experiment result, in which the structure of the alkali films $\mathrm{K}$ and $\mathrm{Cs}$ evolve from the quasihexagonal structure into the (110)-oriented bcc structure.

Using density functional theory (DFT) (Ref. 17) within local-density approximation, Marcus and Allipi ${ }^{12}$ and Alippi et $a l .{ }^{13}$ investigated tetragonal states from the epitaxial strain on (001)-oriented metal films. The epitaxial bain path (EBP), produced by isotropic stress or strain on the (001) plane of the tetragonal phase accompanied by vanishing stress perpendicular to the plane, was defined; stable and metastable phase were found on the EBP, and separated by an unstable region. By means of this, an observed tetragonal state could be identified as a strained form of a particular phase. They studied the EBP of metal materials V, Cu, Co, Ti, Sr, Li, K, and $\mathrm{Rb}$, and compared with experiments.

When materials grow epitaxially on a specified thick substrate, materials will be strained so that its atoms grow in registry with the substrate. If the strain energy is small, the materials are said to be soft with respect to the direction and/or plane; in the opposite case, it is said to be hard. The strain energy is dependent on growth direction and the interaction between the material and substrate (in the present paper, only the effect of the direction is considered). Within the harmonic approximation, ${ }^{7,18-24}$ the softening direction of epitaxial growth is independent of lattice constant of the substrate and definitive, i.e., if [001] is the softest direction, then [111] must be the hardest direction, and vice versa. Ozolinš et $a l .{ }^{6,11}$ have shown that this order does not hold for large deformations. Their calculations illustrated the shift of the softening direction by the anharmonic strain: the softest direction under the biaxial compression is [201] and the hardest direction under the biaxial tension is [110]. The softening under tension along the directions [001] and [111] is attrib- 
uted to the lower energy difference between bcc and fcc phases. With the linear augmented plane wave method (LAPW), Ozolinšs et al. investigated the epitaxial deformation of the noble metals $\mathrm{Cu}, \mathrm{Ni}, \mathrm{Ag}$, and $\mathrm{Au}$. In their works, the softest direction [201] under compression is explained by the loose packing of atoms in the (201) planes.

The adsorption of alkali metal atoms on metal surfaces in the submonolayer has attracted much attention (experimentally and theoretically) in recent years, partly due to the discovery of a variety of new adsorbate phases and technological applications, see Refs. 25-27. Considerably less work in the literature is related to alkali metal films with thicknesses exceeding one monolayer. Recently, Benedek et $a l^{28}$ investigated the growth of a $\mathrm{Na}$ film deposited on the $\mathrm{Cu}(001)$ plane experimentally. Their work revealed that the Na film exhibits a quasihexagonal structure, which gradually evolves with increasing number of layers into (110)-oriented bcc structure. Hulpke et al. ${ }^{29}$ extended the work of Benedek et al. to the other alkali-metal-substrate systems, i.e., $\mathrm{K} / \mathrm{Ni}(001)$ and $\mathrm{Cs} /$ $\mathrm{Cu}(111)$, whose growth behavior is similar to that found by Benedek et al. They found a transition of the alkali metal films from the quasihexagonal structure to the bulk (110)oriented bcc structure occurs more rapidly as one proceeds from $\mathrm{Na}$ to $\mathrm{K}$ and $\mathrm{Cs}$.

This paper is organized as follows. In Sec. II, the calculational formulas are listed. Sec. III gives the equilibrium properties and energy differences of bcc and fcc of alkali metals $\mathrm{K}, \mathrm{Rb}$, and $\mathrm{Cs}$. The results and discussions are presented in Sec. IV. To find the possible metastable states of these alkali metals, both the tensile and compressive epitaxial strain along various directions and/or planes are considered, and the resulting softening sequences are discussed. In Sec. V, we investigate the structural transition of alkali metal films from hcp to bcc. The conclusions are given in the last section.

\section{COMPUTATIONAL FRAME}

\section{A. Epitaxial strain energy and softening function}

In this section, formulas that are used in present work are given. As shown in Ref. 6, some assumptions are necessary. First, the film, being much thinner than the substrate, maintains coherency with the substrate and plastically deforms to accommodate the lattice mismatch at the interface. Furthermore, the film is thick enough so that the chemical interaction energy at the film-substrate interface and the filmvacuum surface is negligibly small in comparison to the elastic deformation energy of the film. (By means of this, our calculations are implemented by a strained bulk without the substrate. It is more realistic to do with a strained slab, as suggested in Ref. 13.) The effect of other imperfections, e.g., surface steps, terraces, impurities, and inhomogeneous strained fields, are excluded. We consider a film coherently strained on a substrate oriented along the direction $\hat{G}$ with the surface unit cell vectors $\mathbf{a}_{1}$ and $\mathbf{a}_{2}$, orthogonal to $\hat{G}$. The epitaxial strain energy $E^{\text {epi }}\left(\mathbf{a}_{1}, \mathbf{a}_{2}, \hat{G}\right)$ is the strain energy deformed in the growth plane with the unit cell vectors $\left\{\mathbf{a}_{1}, \mathbf{a}_{2}\right\}$ of the substrate, and relaxed with respect to the out-of-plane vectors $\mathbf{c}$ :

$$
E^{\mathrm{epi}}\left(\mathbf{a}_{1}, \mathbf{a}_{2}, \hat{G}\right)=\min _{\mathbf{c}}\left[E^{\mathrm{tot}}\left(\mathbf{a}_{1}, \mathbf{a}_{2}, \mathbf{c}\right)\right]-E^{\mathrm{tot}}\left(a_{e q}\right) .
$$

Here, $\mathbf{a}_{1}$ and $\mathbf{a}_{2}$ are determined by the following relation:

$$
\mathbf{a}_{i}=\left(\frac{a_{s}}{a_{e q}}\right) \mathbf{a}_{i}^{0}, \quad i=1,2,
$$

where $a_{s}$ and $a_{e q}$ are lattice parameters of the substrate and the unstrained film, respectively, and $\mathbf{a}_{1}^{0}$ and $\mathbf{a}_{2}^{0}$ are the surface unit vectors of the unstrained film. So the epitaxial strain energy becomes a function of strain and direction $\hat{G}$ only:

$$
E^{\mathrm{epi}}\left[\left(a_{s} / a_{e q}\right) \mathbf{a}_{1}^{0},\left(a_{s} / a_{e q}\right) \mathbf{a}_{2}^{0}, \hat{G}\right] \equiv E^{\mathrm{epi}}\left(a_{s}, \hat{G}\right) \equiv E^{\mathrm{epi}}(\varepsilon, \hat{G}),
$$

where $\varepsilon=\left(a_{s}-a_{e q}\right) / a_{e q}$.

The softening function is defined as

$$
q(\hat{G}, \varepsilon)=\frac{E^{\mathrm{epi}}(\varepsilon, \hat{G})}{E^{\mathrm{bulk}}(\varepsilon)},
$$

where $E^{\text {bulk }}(\varepsilon)=E^{\text {tot }}\left(a_{s}\right)-E\left(a_{e q}\right)$ is the strain energy under hydrostatic pressure. For harmonic strain both $E^{\mathrm{epi}}(\varepsilon, \hat{G})$ and $E^{\text {bulk }}(\varepsilon)$ are linear functions of the strain squared, and the resulting $q_{\text {harm }}(\hat{G})^{7}$ is

$$
q_{\text {harm }}(\hat{G})=1-\frac{B}{C_{11}+\Delta \gamma_{\text {harm }}(\hat{G})},
$$

where $\quad B=\frac{1}{3}\left(C_{11}+2 C_{12}\right), \quad \Delta=C_{44}-\frac{1}{2}\left(C_{11}-C_{12}\right), \quad$ and $\gamma_{\text {harm }}(\hat{G})$ is a geometric function of the spherical angles formed by $\hat{G}$. From the above equation, we see that the softening function is independent of the lattice constant, and the softest and hardest directions are definitive. If deformation of the substrate is included, the softening function will depend on the strain energy of the substrate and interface energy, and a simple analytic formula will not exist.

In our calculations, we find that the equilibrium fcc structure of alkali metals is the energetically most favorable at absolute zero and low pressure, compared to the bcc and hcp structures, and is selected as the initial unstrained structure.

\section{B. $a b$ initio calculations}

The total energy calculations are implemented by using the $a b$ initio pseudopotential plane wave method, ${ }^{30}$ and the pseudopotentials are generated by the Hamann approach ${ }^{31,32}$ within the local-density approximation of Perdew and Zunger. ${ }^{33}$ The cutoff energy of $\mathrm{K}, \mathrm{Rb}$, and $\mathrm{Cs}$ are 7,7 , and 6 Ry, respectively. An electronic temperature is introduced for the following purposes: (1) to improve the Brillouin zone sampling and (2) to reduce oscillations of the electron density by decreasing the amount of charge which flows between the states near Fermi level as the electronic structure evolves in the self-consistent loop. Here, the electronic temperature is set $0.3 \mathrm{eV}$, and the total energy is reduced to absolute zero by approach of Gillan. ${ }^{34}$ With these parameters, our $k$-space mesh is $12 \times 12 \times 12$ for four atoms in a fcc supercell at the equilibrium constant, and the total energy changed less than $\sim 0.01 \mathrm{mRy}$. To keep the calculations un- 
TABLE I. Equilibrium properties of $\mathrm{K}, \mathrm{Rb}$, and $\mathrm{Cs}$. Comparison with previous first principles calculations and experimental data are also given. The left column is for bcc, and the right column is for fcc. The unit length is Bohr, moduli is GPa, the energy difference per atom between bcc and fcc, $\Delta E=E_{\mathrm{bcc}}-E_{\mathrm{fcc}}$, is in mRy. Exp. means experimental results.

\begin{tabular}{lccccccccc}
\hline \hline & $a_{0}$ & $C_{11}$ & $C_{12}$ & $C_{44}$ & $a_{0}$ & $C_{11}$ & $C_{12}$ & $C_{44}$ & $\Delta E$ \\
\hline $\mathrm{K}^{\mathrm{a}}$ & 9.65 & 3.59 & 2.87 & 2.98 & 12.17 & 4.07 & 3.19 & 2.97 & 0.123 \\
$\mathrm{~K}^{\mathrm{b}}$ & 9.50 & 6.1 & 4.9 & & 11.99 & 5.7 & 5.0 & & 0.02 \\
$\mathrm{~K}($ Exp.) & $9.87^{\mathrm{d}}$ & $4.16^{\mathrm{c}}$ & $3.4^{\mathrm{c}}$ & $2.86^{\mathrm{c}}$ & & & & & \\
$\mathrm{Rb}^{\mathrm{a}}$ & 10.43 & 2.99 & 2.55 & 2.10 & 13.15 & 3.35 & 2.68 & 1.98 & 0.122 \\
$\mathrm{Rb}^{\mathrm{b}}$ & 10.129 & 4.5 & 3.8 & & 12.756 & 4.6 & 4.0 & & 0.17 \\
$\mathrm{Rb}$ (Exp.) & $10.55^{\mathrm{d}}$ & $3.42^{\mathrm{c}}$ & $2.88^{\mathrm{c}}$ & $2.21^{\mathrm{c}}$ & & & & & \\
$\mathrm{Cs}$ & & & & & & & & & \\
$\mathrm{Cs}$ (Exp.) & 11.54 & 2.10 & 1.83 & 1.48 & 14.55 & 2.34 & 1.84 & 1.36 & 0.182 \\
\hline \hline
\end{tabular}

${ }^{\mathrm{a} P r e s e n t}$ work.

${ }^{\mathrm{b}}$ Reference 35 with WIEN95.

${ }^{c}$ Elastic constants at $T=4.2 \mathrm{~K}$ (Ref. 36).

dbcc lattice constants at $T=5 \mathrm{~K}$ (Ref. 37).

der the same accuracy, the number of $k$ points per unit length of deformed structures is held constants approximately.

\section{EQUILIBRIUM PROPERTIES OF ALKALI METALS}

The equilibrium properties of $\mathrm{K}, \mathrm{Rb}$, and $\mathrm{Cs}$ with bcc and fcc structures are calculated and are shown in Table I. The experimental data listed are from the stable and low temperature phases. Agreement between experiment and theory is good. For comparison, we also calculate the equilibrium properties of alkali metals within the generalized gradient approximation (GGA) and considered the nonlinear interaction of core-valence exchange correlation. Although it has been shown that the GGA and the nonlinear core correction is necessary for alkali metal atoms, ${ }^{38}$ we find that, the resulting elastic moduli is too small, for example, $\mathrm{C}_{11}$ of $\mathrm{K}: 3.03$ $\mathrm{GPa}(\mathrm{bcc})$ and $3.38 \mathrm{GPa}$ (fcc), compared to the results obtained using the LDA of $3.59 \mathrm{GPa}(\mathrm{bcc})$ and $4.07 \mathrm{GPa}$ (fcc), and experimental value, $4.16 \mathrm{GPa}$. We therefore chose to implement the LDA throughout the paper. As shown in Table I, both bcc and fcc are structural stable, where the fcc phase is energetically favorable. These trend agree well with the previous full potential linear augmented plane wave (FPLAPW) calculations. ${ }^{35}$

\section{EPITAXIAL DEFORMATION OF ALKALI METAL}

\section{A. Metastable state}

In this section, epitaxial deformations of alkali metals, $\mathrm{K}$, $\mathrm{Rb}$, and $\mathrm{Cs}$, subjected to an isotropic expansion and compression on the (001), (110), (111), and (201) planes, are investigated. The results for the (001) case are shown in Fig. 1. During deformation, tetragonal symmetry is held, and the extrema is approached at the state with higher symmetry. When the ratio of c/a decreases from the initial value 1 to $1 / \sqrt{2}$ by the biaxial expansion of the (001) plane, the structure transforms from fcc to bcc. The variations of longitudi-

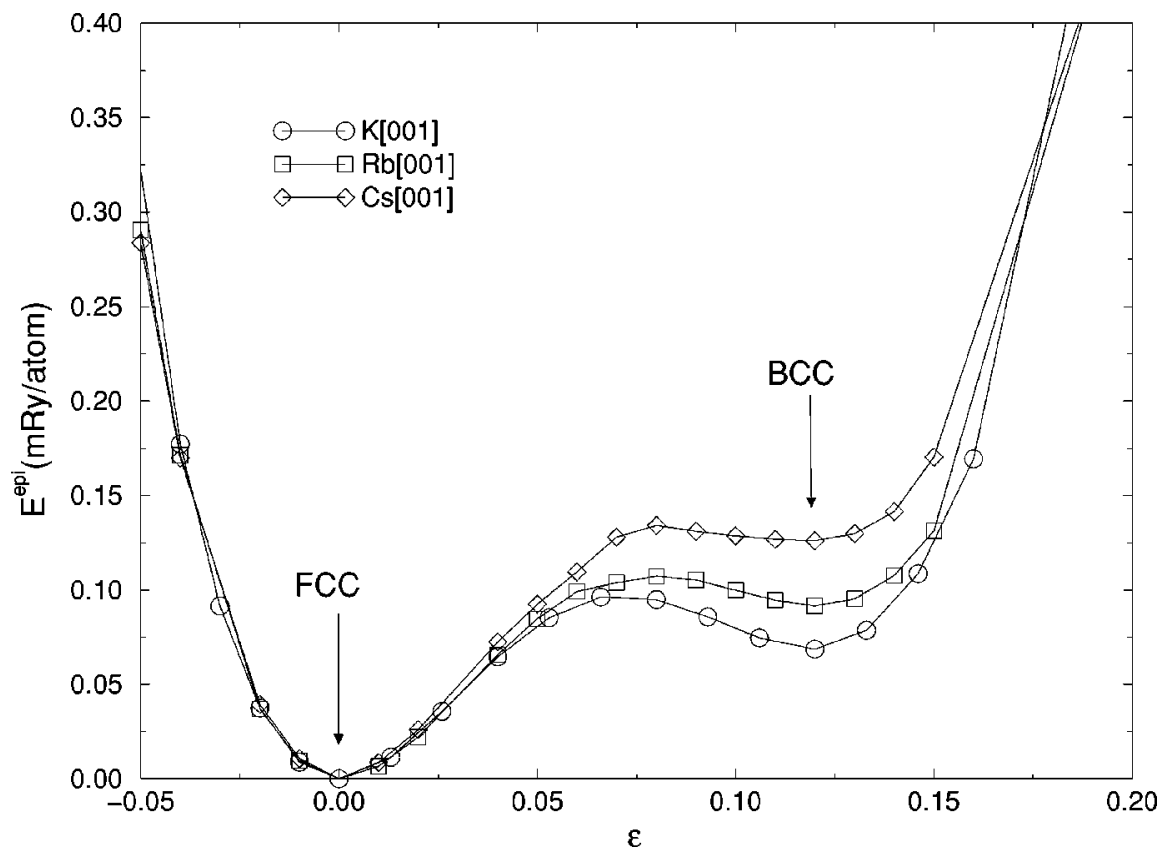

FIG. 1. The epitaxial strain energy of $\mathrm{K}, \mathrm{Rb}$, and $\mathrm{Cs}$ along the direction [001]. Two phases with fcc (stable) and bcc (metastable) structure, separated by a local maximum with tetragonal symmetry, are found. 


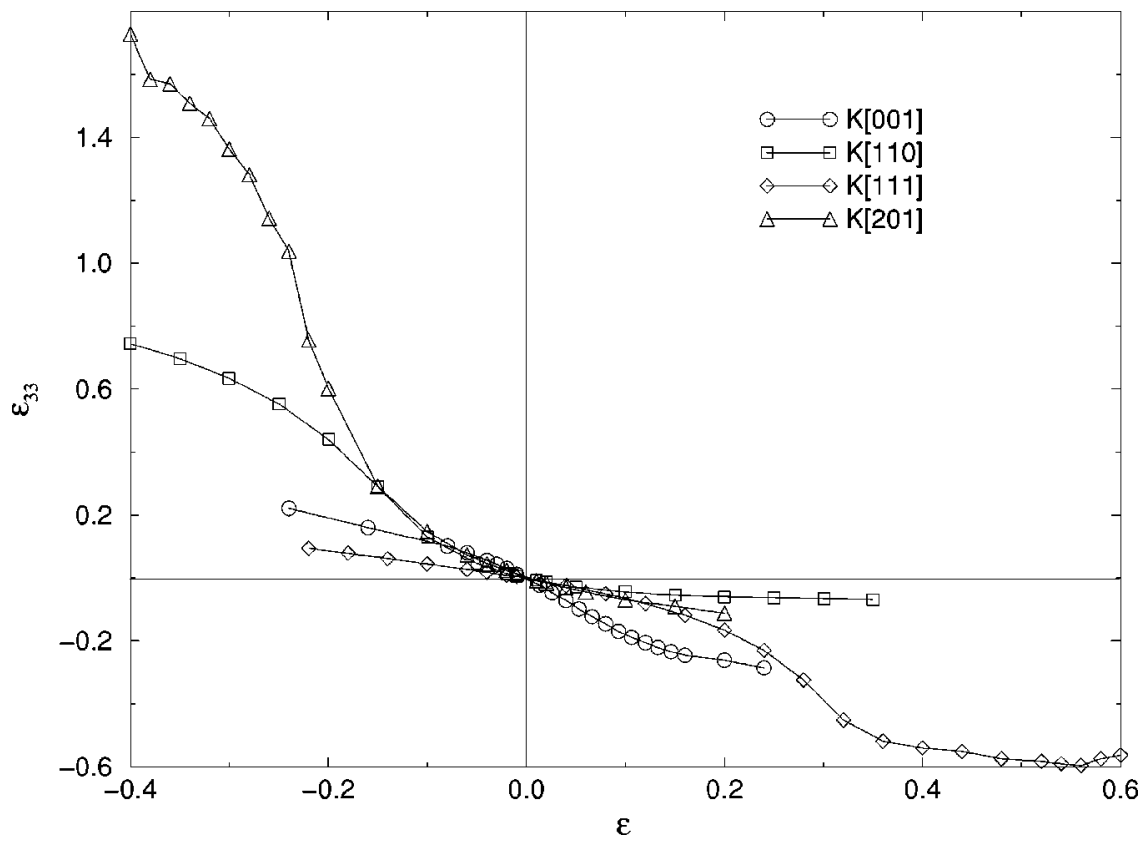

FIG. 2. The variation of the longitudinal strain versus the transverse strain of alkali metal $\mathrm{K}$ along various directions. Referring this figure, the structural parameter of strained structure can be obtained. The appearance of $\mathrm{Rb}$ and $\mathrm{Cs}$ is similar.

nal strain of $\mathrm{K}$ under the various directions and/or planes versus the transverse strain are shown in Fig. 2; the appearance of $\mathrm{Rb}$ and $\mathrm{Cs}$ is similar, and are not presented. As shown in Sec. III, both the fcc and bcc states of $\mathrm{K}, \mathrm{Rb}$, and $\mathrm{Cs}$ are structural stable at absolute zero, and will stay at the local minimum. Referring to the structural parameters given by Fig. 2, the right local minimum in Fig. 1 can be identified as the bcc structure, and for $\mathrm{Rb}$ and $\mathrm{Cs}$, the conclusion is same. Between these two minima, there exists a local maximum without higher symmetry than the tetragonal structure, as shown in Fig. 1. Since both the fcc and bcc states are free of stress and are located at a local minimum, they are the phase of the materials, and the bcc state is a metastable phase due to its higher energy. Under biaxial compression, there are no metastable states. It is worth mentioning that the responses of the three alkali metals under the epitaxial strain at absolute zero are similar, and this feature is held for other directions.
The materials under the loading on the (110) plane show no metastable state, as shown in Fig. 3, this is because no higher symmetry than the initial structure occurs. In the case of the (111) plane, trigonal symmetry is held throughout the deformation path. The value of c/a ratio, equal to $\sqrt{6}$ at the initial state, decreases and goes through the state $\sqrt{6} / 2$ and $\sqrt{6} / 4$ under the biaxial tension. These two special values just correspond to the simple cubic (sc) structure and the bcc structure, by some geometric calculations. Owing to their higher symmetry, it must be located at the extrema, respectively. Concerning Figs. 4 and 2, the local maximum can be identified as the sc structure, and the right local minimum as the bcc structure, presented as a metastable state for its higher energy than the fcc phase, respectively. The energy barrier (note the change in the energy scale compared to Figs. 1 and 3) is very big for the large difference of structure parameters of these two phases evolved on the epitaxial path.

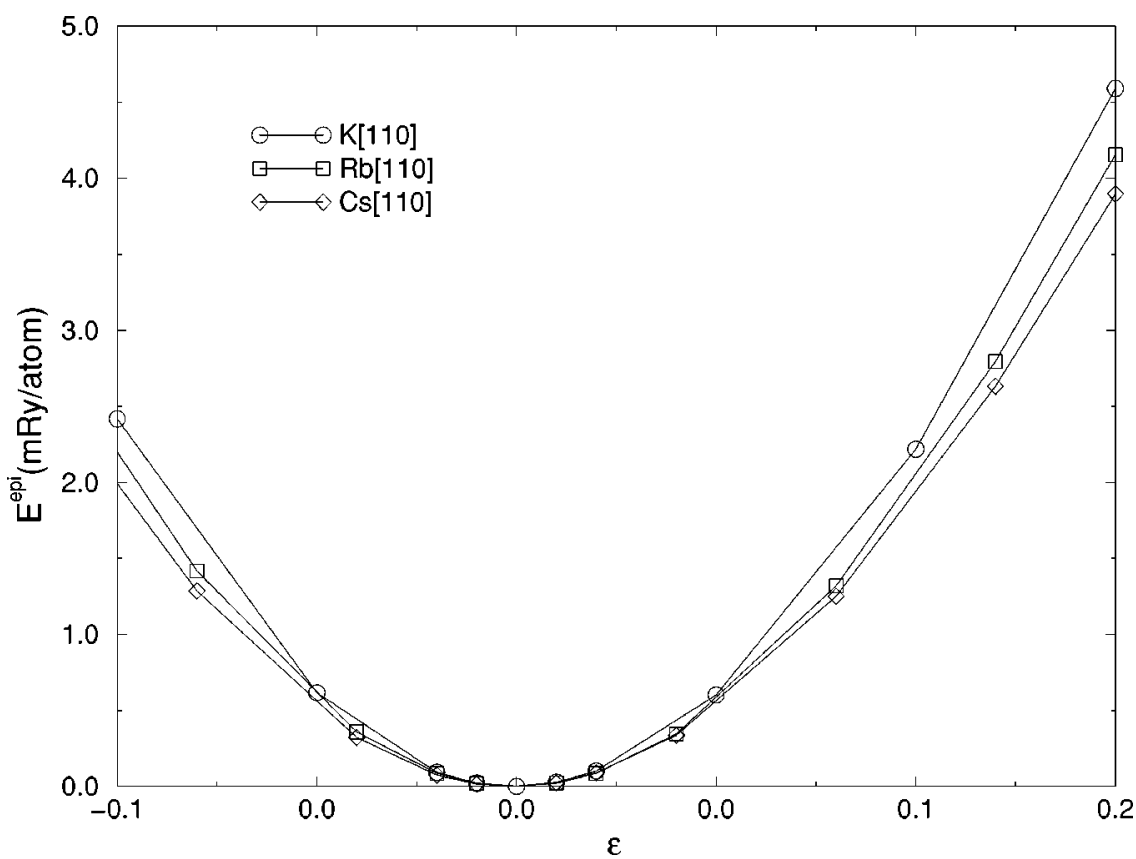

FIG. 3. The epitaxial strain energy of $\mathrm{K}, \mathrm{Rb}$, and $\mathrm{Cs}$ along the direction [110]. The metastable state are not found throughout the configurational space. 


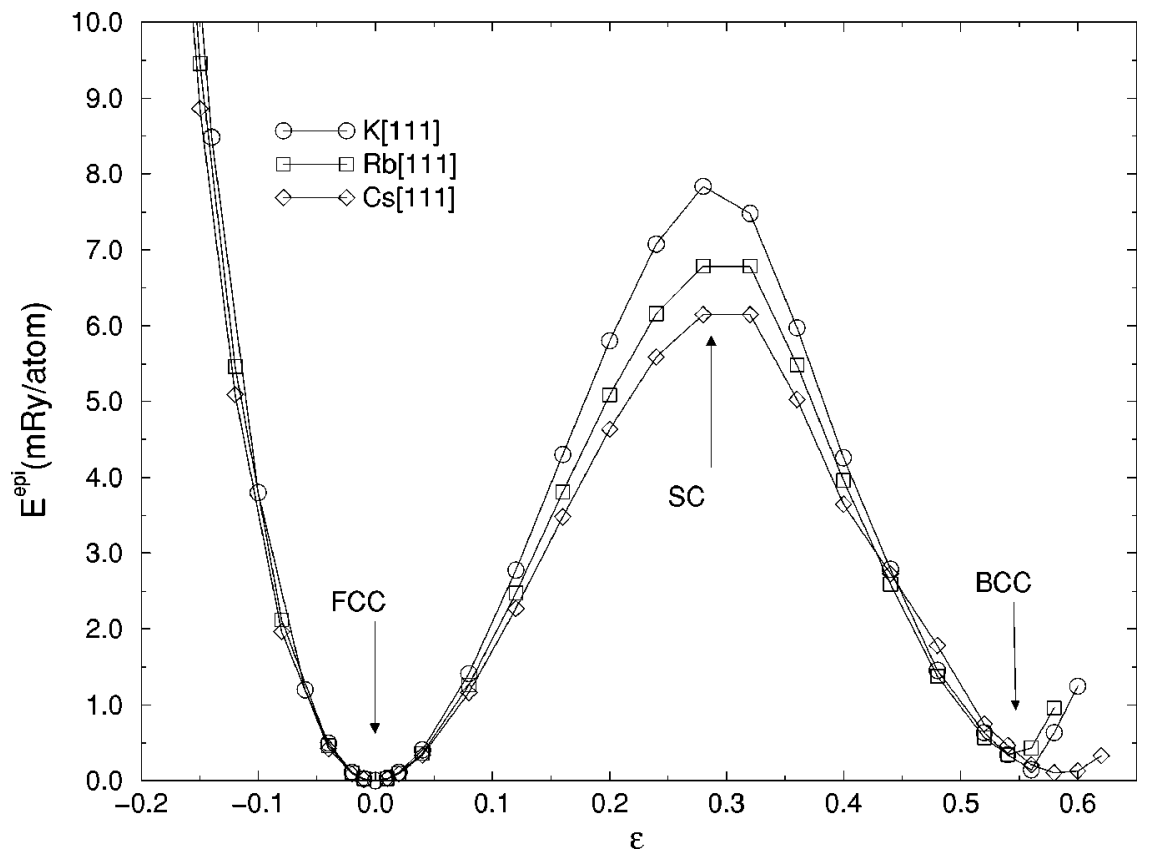

FIG. 4. The epitaxial strain energy of $\mathrm{K}, \mathrm{Rb}$, and $\mathrm{Cs}$ along the direction [111]. Two phases with fcc (stable) and bcc (metastable), separated by a local maximum with simple cubic (sc) structure, are found. Note the change in the energy scale compared to the Fig. 1 .

With increase of the atomic number, the energy barrier decreases. Just like the deformation on the (001) plane, there does not exist a metastable state under the compression.

When the materials grow on the (201) plane, the case is somewhat more complicated, and its initial unstrained (010) section is shown in Fig. 5. Lattice vectors of the supercell are $\mathbf{a}_{1}=[010], \mathbf{a}_{2}=-[100]+2[001], \mathbf{a}_{3}=2[100]+[001]$ (the unit is $a_{s}$ ), respectively. The supercell contains twenty atoms: ten atoms are shown in Fig. 5 (black circles), and the remaining atoms can be obtained by a displacement vector $(0.5,0.5,0) a_{s}$. The epitaxial strain energy of $\mathrm{K}, \mathrm{Rb}$, and $\mathrm{Cs}$ on this plane are shown in Fig. 6. From this figure, we find, under the biaxial compressive strain, a local minimum is present, and one metastable state occurs. The corresponding structural parameters, e.g., K, can be obtained by referring to Figs. 6 and 2. Considering Fig. 5 and the structural parameters obtained, we know, the symmetry of the resulting structure is very low; no simple structure can be identified, and we call it a "superstructure", of alkali metals. This "superstructure" is approached after suffering a large structural transformation, and the corresponding energy barrier, which decreases with increase of the atomic number, is huge, compared to Fig. 1. No metastable state is found under biaxial tension.

\section{B. The softening direction}

Based on the above results, the softening function of $\mathrm{K}$, $\mathrm{Rb}$, and $\mathrm{Cs}$ are calculated. Due to the similarity of the alkali metals considered under the epitaxial deformation at the absolute zero, only the results for $\mathrm{K}$ are presented, and the conclusions obtained can likely be applied to $\mathrm{Rb}$ and $\mathrm{Cs}$. The epitaxial strain energy on the plane (001), (110), (111), and (201) and the strain energy under the hydrostatic pressure are plotted in Fig. 7(a); the softening functions $\mathrm{q}(\hat{G}, \varepsilon)$ calculated are given in Fig. 7(b).

At the small strain, as shown in Fig. 7(b), the softest direction is [001], while the hardest direction is [111]; the magnitudes of the softening function along the directions
[110] and [201] are intermediate. These results agree with the traditional harmonic theory. However, with increase of the strain, the softening functions begin to cross and become dependent on the lattice constant of the substrate significantly; the sequence of the softening functions shifts, respectively. Because, under the expansive strain along the directions [001] and [111] (as shown in above calculations), there exist metastable states, and the growth of $\mathrm{K}, \mathrm{Rb}$, and $\mathrm{Cs}$ in these two cases are softened. Furthermore, the direction [001] becomes the softest direction due to its smaller energy barrier than that of the [111], and this still agrees with the harmonic theory. The growth along the directions [110] and [201] under the expansion becomes difficult due to the absence of a metastable state. Because the (201) plane is a loose packing plane compared to the (110) plane, it is ener-

[201]

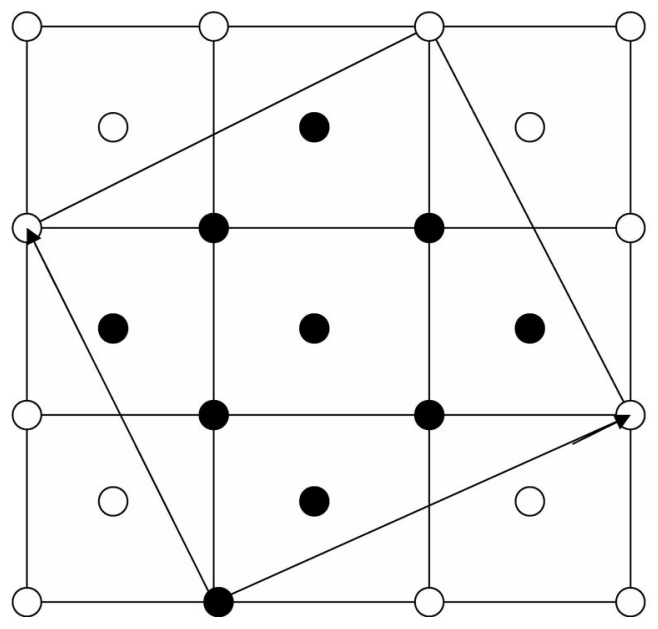

$[-102]$

FIG. 5. The initial unstrained structure model when the materials grow along the direction [201]. Lattice vectors of supercell are: $\mathbf{a}_{1}=[010], \quad \mathbf{a}_{2}=-[100]+2[001], \quad \mathbf{a}_{3}=2[100]+[001], \quad$ the unit is $a_{s}$. The plane shown in the figure is (010) plane. The supercell contains twenty atoms: ten atoms are shown in the figure (black circles), and the remaining can be obtained by a displacement vector $(0.5,0.5,0) a_{s}$. 


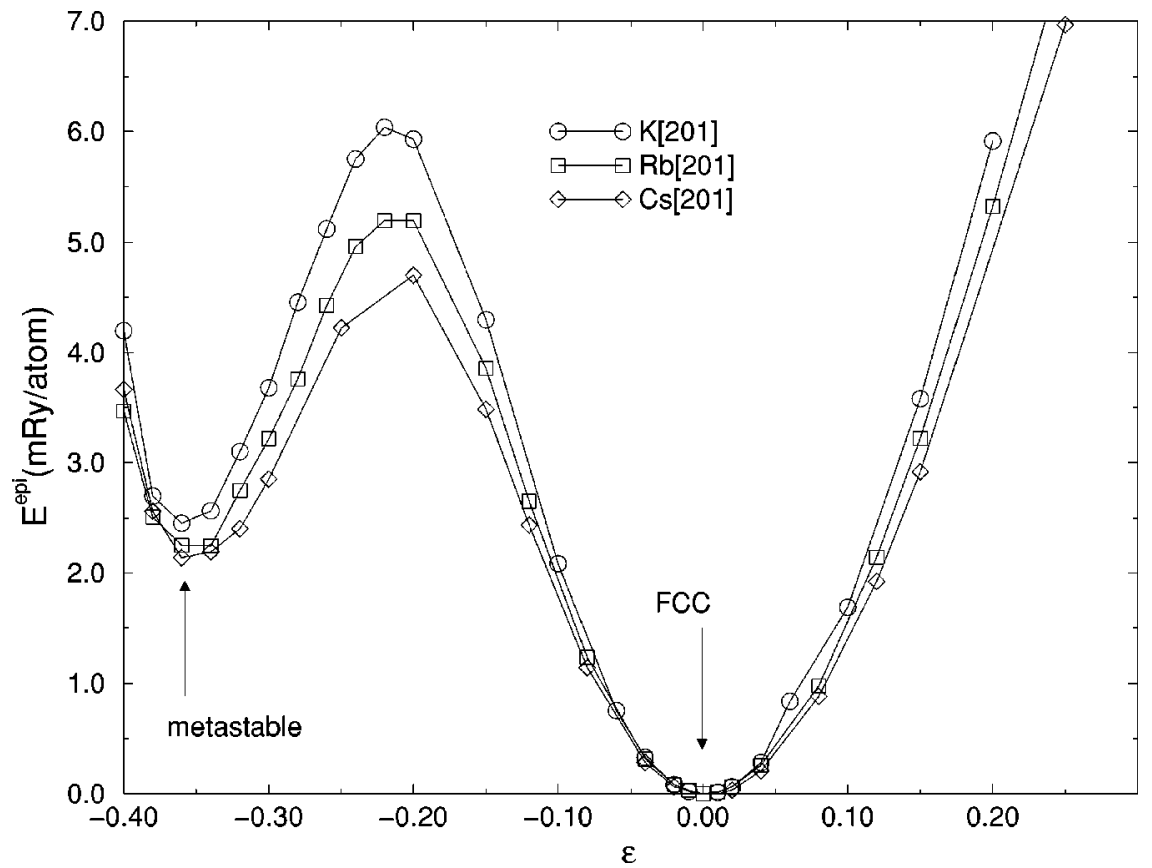

FIG. 6. The epitaxial strain energy of $\mathrm{K}, \mathrm{Rb}$, and $\mathrm{Cs}$ along the direction [201]. Two phases with fcc (stable) and superstructure (metastable) without any simple structure, separated by a local maximum, are found. Note the change in the energy scale compared to the Fig. 1.

getically favorable. This makes the growth along the direction [110] the hardest direction, instead of the direction [111], as predicted by the harmonic theory.

From Fig. 7(b), we see that the softest direction under the large biaxial compression is [201], instead of [001] as given by the harmonic theory. This fact illustrates sufficiently strong dependence of the epitaxial growth on the existence of the metastable state. However, the hardest direction is still [111], the same as obtained with the harmonic theory. Although Ozolinšs et al. ${ }^{6}$ had found that the direction [201] is the softest direction under the biaxial compression for noble metals, the metastable state found here was not explicitly shown in their papers, and they attributed the softening to the comparable loose packing in the (201) planes.

\section{V. hep TO bec}

As mentioned in the Sec. I, Na films can be deposited on the $\mathrm{Cu}(001)$ plane, and the initial structure is a quasihexagonal structure. With increase of the number of the layers, the quasihexagonal structure distorts and evolves into a (110)oriented bcc structure. A similar phenomenon is also found in the $\mathrm{K} / \mathrm{Ni}(001)$ and $\mathrm{Cs} / \mathrm{Cu}(111)$ systems. Despite the difference of crystallographic plane of the substrates, the resulting structure is the same.

Recently, Craievich et al. ${ }^{16}$ investigated the hcp $\rightarrow$ bcc transformation of transitional metals via first principles total energy calculations at finite electronic temperature, and their method can be used to investigate the above experimental phenomena. In this method, a orthorhombic two-atom unit cell with the lattice constants $a, b$, and $c$ is defined. The atoms are at $(0,0,0)$ and $(\xi a, 0, c / 2)$. Both of the hcp and bcc structures can be included in this supercell. The relationship between the orthorhombic parameters and the conventional cells are for hcp structure, $a=\sqrt{3} a_{\mathrm{hcp}}, b=a_{\mathrm{hcp}}, c=c_{\mathrm{hcp}}$, and $\xi=1 / 3$; for bcc structure, $a=c=\sqrt{2} a_{\mathrm{bcc}}, b=a_{\mathrm{bcc}}$, and $\xi=1 / 2$. To require the distance of the nearest and nextnearest neighbors in and out of plane equal and volume con- serving, a set of a single-parameter control equations are obtained as shown in the following:

$$
\begin{gathered}
\frac{c^{2}}{a^{2}}=4 \xi(1-\xi), \\
\frac{b^{2}}{a^{2}}=\xi, \\
a^{3}=\frac{\Omega}{\xi(1-\xi)^{1 / 2}},
\end{gathered}
$$

where $\Omega=(1 / 2) a b c$. The lattice constants $a, b$, and $c$ are determined in terms of the single parameter $\xi$. When $\xi$ $=1 / 3$, it corresponds to the hexagonal in-plane ordering

$$
\left(\frac{c}{a}\right)^{2}=\frac{8}{9}=\frac{1}{3}\left(\frac{c}{a}\right)_{\mathrm{hcp}}^{2},
$$

i.e., ideal hcp $c / a$ ratio. Similar, for $\xi=1 / 2$, the correct bcc structure is obtained. In this supercell, the plane constructed by lattice vectors $a$ and $b$ in the case of $\xi=1 / 3$ is just the (0001) plane of hcp with hexagonal symmetry, and $\xi=1 / 2$, (110) plane of bcc structure, respectively. ${ }^{16,39}$

Our results for $\mathrm{K}, \mathrm{Rb}$, and $\mathrm{Cs}$ at the absolute zero are given in Fig. 8, and the path of the structural transition from hcp to bcc is built. For higher symmetry in the hcp and bcc structures along this path, the corresponding state should be an extrema. ${ }^{16}$ From Fig. 8, we find that both of hcp and bcc are in a local minimum, and hcp structure is also one of the phase of the alkali metals considered. This is different from the Craievich's results, ${ }^{16}$ in which the bcc state of the transitional metals $\mathrm{Nb}$ and Mo stayed at the local minimum, and the hcp state at the local maximum, i.e., an unstable state. The energy difference per atom between the bcc and hcp phase, i.e., $E_{\mathrm{bcc}}-E_{\mathrm{hcp}}$, is $0.062 \mathrm{mRy}$ for $\mathrm{K}, 0.115 \mathrm{mRy}$ for $\mathrm{Rb}$, and $0.121 \mathrm{mRy}$ for $\mathrm{Cs}$. The resulting energy difference 

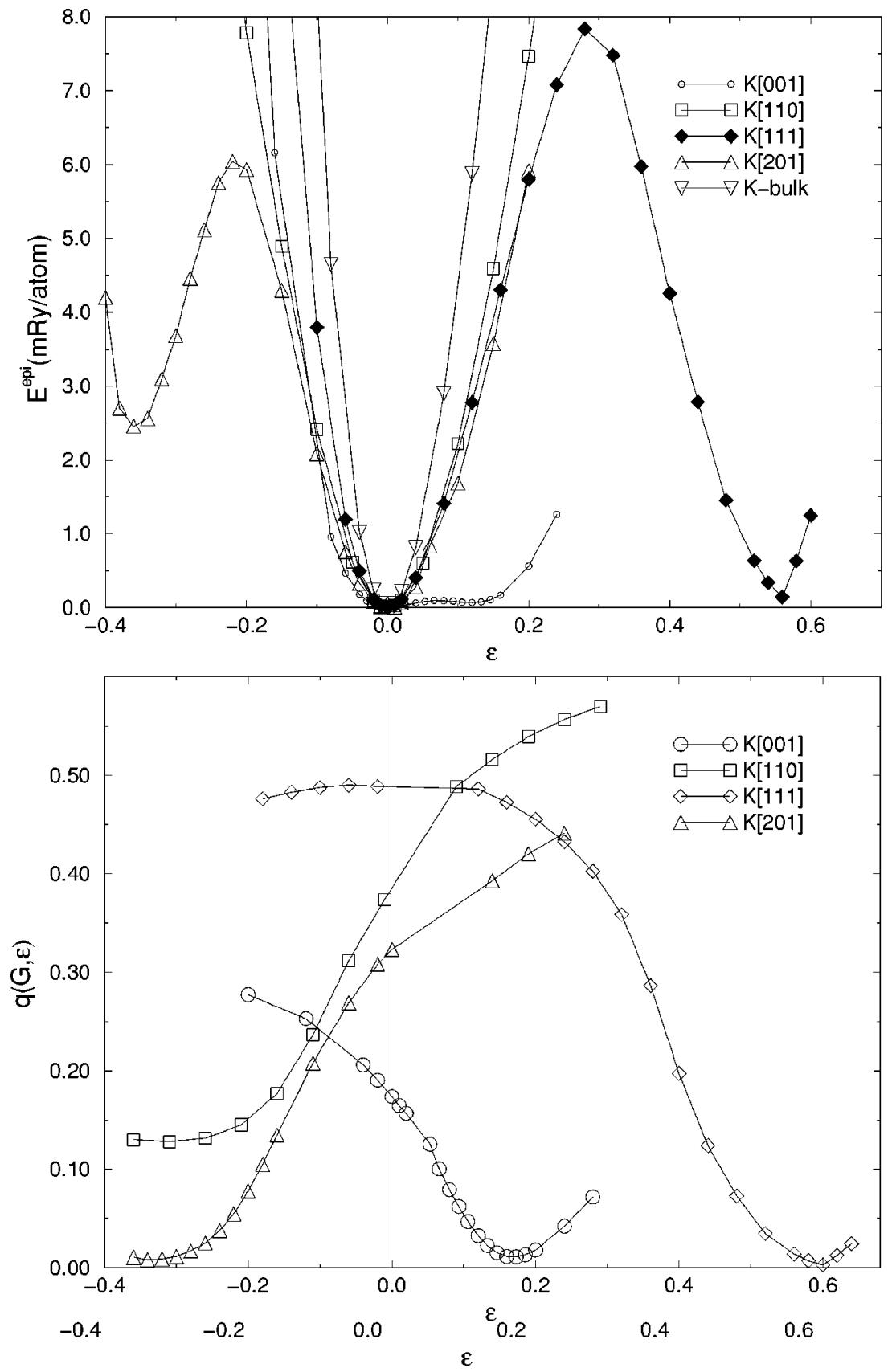

FIG. 7. The epitaxial strain energy (a) and softening function (b) of $\mathrm{K}$ along the directions [001], [110], [111], and [201]. Here, bulk means the strain energy under the hydrostatic pressure.

per atom between hcp and fcc, $E_{\mathrm{hcp}}-E_{\mathrm{fcc}}$ is $0.06 \mathrm{mRy}$ for $\mathrm{K}$, $0.007 \mathrm{mRy}$ for $\mathrm{Rb}$ (almost no difference), and $0.06 \mathrm{mRy}$ for $\mathrm{Cs}$, respectively. The resulting energy sequence of alkali metals $\mathrm{K}, \mathrm{Rb}$, and $\mathrm{Cs}$ from high to low is bcc $\rightarrow$ hcp $\rightarrow$ fcc at absolute zero temperature and low pressure, which agree with the previous $a b$ initio calculations. ${ }^{40}$ hcp and bcc structures are connected by an orthorhombic deformation path.

In the experiments of Hulpke et al. ${ }^{29}$ it was found that the transition of the alkali metal films from the quasihexagonal structure to the bulk (110)-oriented bcc structure occurs more rapidly as one proceeds from $\mathrm{Na}$ to $\mathrm{K}$ and $\mathrm{Cs}$. However, as shown in Fig. 8, energy barrier from hcp to bcc increases from $\mathrm{K}$ to $\mathrm{Rb}$ and $\mathrm{Cs}$, while the depth of the potential well of the bcc state becomes shallower, i.e., with increase of the atomic number, the transition becomes more difficult and the resulting structure destabilized. On the other hand, the hcp structure is always energetically more favorable than the bcc structure, in present calculations, i.e., at absolute zero temperature and low pressure, and the structural transition from hcp to bcc will not be expected. To resolve these questions one should consider the effect of thermodynamics, ${ }^{16,41,42}$ and the relative stability of the various phases at finite temperatures as determined by the Gibbs free energy, which includes the contribution from the pressure-volume term, vibration entropy and electronic entropy. We also note that the energy difference, typically $0.1 \mathrm{mRy}$, between hcp and bcc of three alkali metals is comparatively small. Small disturbances, e.g., vibration entropy, the pressure-volume term, the effect of the interface, or imperfection of the film can change the relative stability of these two phases completely. Actually, a terrace size of at least several $100 \AA$ cannot be excluded for all the substrates in the experimental samples, ${ }^{29}$ and strain, introduced by the lattice mismatch at the substrate-film interface, is gradually removed during the growth process. 


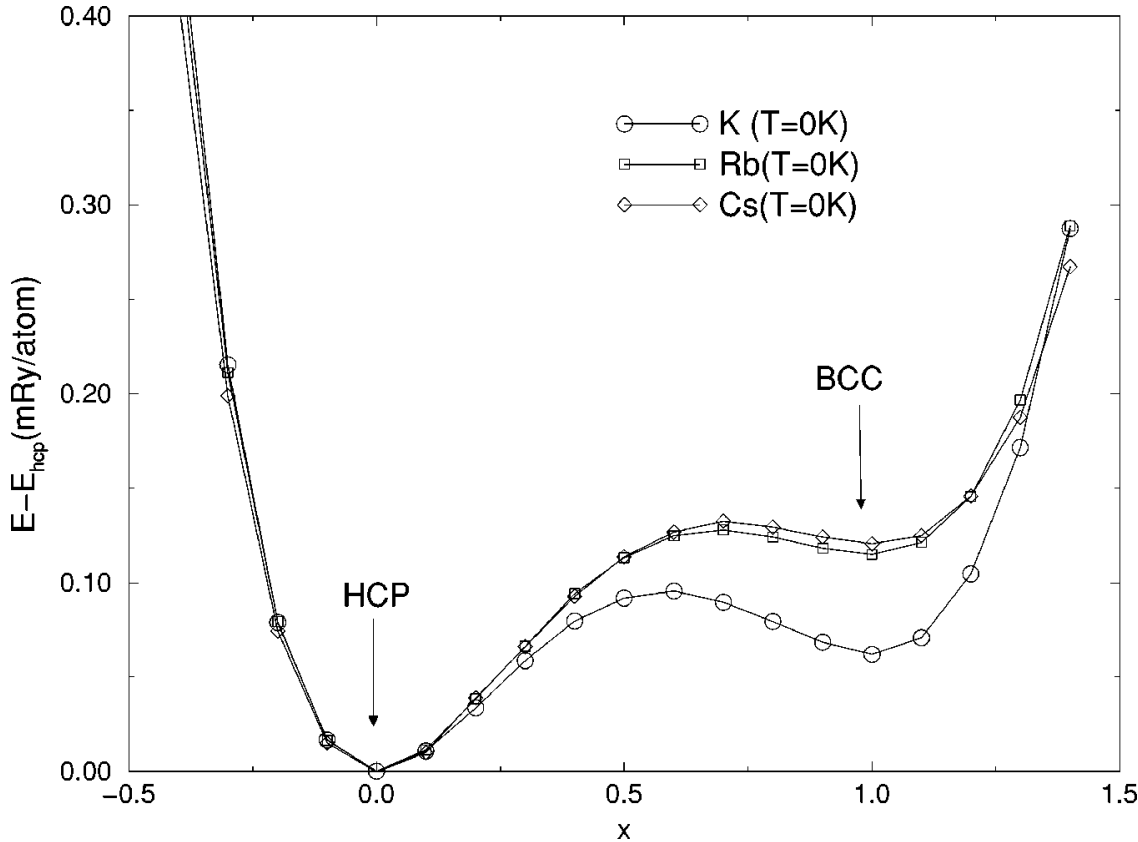

FIG. 8. The total energy along the orthorhombic deformation path connecting hep and bcc for $\mathrm{K}, \mathrm{Rb}$, and $\mathrm{Cs}$ at absolute zero $(x=6 \xi-2)$.

\section{CONCLUSIONS}

The epitaxial deformation of the alkali metals $\mathrm{K}, \mathrm{Rb}$, and Cs on the various planes, i.e., (001), (110), (111), and (201), has been investigated. Metastable states, besides the fcc phase, are identified except for the case of (110) plane. The fcc structure is always the stable and energetically favorable phase. The metastable bcc structure has been found by biaxial tension on the (001) and (111) planes and a metastable state without any simple structure is obtained by biaxial compression when the materials deform on the (201) plane.

The existence of the metastable states and the magnitude of the energy barriers significantly affect the softening direction and the corresponding softening sequence of epitaxial deformation of the materials. The existence of the bcc phase under biaxial tension in the [001] and [111] directions results in softening in these two directions. Owing to the smaller energy barrier in the [001] direction, compared to the [111] direction, it becomes the softest direction. For the loose atomic packing in the (201) planes, the deformations on this plane under biaxial tension becomes the hardest. When biaxial compression is loaded on the materials, the [201] direc- tion becomes the softest direction due to the existence of the metastable states. The resulting sequence of the softening function from soft to hard is, under biaxial compression, [201], [110], [001], and [111]; under biaxial tension, [001], [111], [201], and [110]. At the absolute zero temperature, the response of the alkali metals $\mathrm{K}, \mathrm{Rb}$, and $\mathrm{Cs}$ versus the epitaxial strain in the various planes are similar.

An orthorhombic deformation path is used to investigate the fact, that the structure of the alkali films $\mathrm{K}$ and $\mathrm{Cs}$ evolves from the quasihexagonal structure into the (110)oriented bcc structure, as observed by experiments. However, further work, concerning the effect of thermodynamics, the interface and imperfections, is necessary.

\section{ACKNOWLEDGMENTS}

This work was supported by the National Natural Science Foundation of China (Grant No. 19704100) and National Natural Science Foundation of Chinese Academy of Science (Grant No. KJ951-1-201). We also thank Dr. C. Stampfl for reading the manuscript.
*Electronic address: wxli@fhi-berlin.mpg.de

${ }^{1}$ C. Günther, J. Vrijmoeth, R. Q. Hwang, and R. J. Behm, Phys. Rev. Lett. 74, 754 (1995).

${ }^{2}$ R. Q. Hwang, J. C. Hamilton, J. L. Stevens, and S. M. Foiles, Phys. Rev. Lett. 75, 4242 (1995).

${ }^{3}$ G. Vidali and H. Zeng, Appl. Surf. Sci. 92, 11 (1996).

${ }^{4}$ J. H. van der Merwe, CRC Crit. Rev. Solid State Mater. Sci. 17, 187 (1991).

${ }^{5}$ J. C. Hamilton and S. M. Foiles, Phys. Rev. Lett. 75, 882 (1995).

${ }^{6}$ V. Ozolinšs, C. Wolverton, and A. Zunger, Phys. Rev. B 57, 4816 (1998).

${ }^{7}$ A. Zunger, in Handbook of Crystal Growth, edited by D. T. J. Hurle (Elsevier, Amsterdam, 1994), Vol. 3, p. 997, and references therein.
${ }^{8}$ C. P. Wang, S. C. Wu, F. Jona, and P. M. Marcus, Phys. Rev. B 49, 17385 (1994).

${ }^{9}$ A. A. Saleh, V. Shutthanandan, and R. J. Smith, Phys. Rev. B 49, 4908 (1994).

${ }^{10}$ H. Wormeester, E. Hüger, and E. Bauer, Phys. Rev. Lett. 77, 1540 (1996)

${ }^{11}$ V. Ozoliņš, C. Wolverton, and A. Zunger, Appl. Phys. Lett. 72, 427 (1998).

${ }^{12}$ P. M. Marcus and P. Alippi, Phys. Rev. B 57, 1971 (1998).

${ }^{13}$ P. Alippi, P. M. Marcus, and M. Scheffler, Phys. Rev. Lett. 78, 3892 (1997).

${ }^{14}$ F. Milstein, H. Fang, and J. Marschall, Philos. Mag. A 70, 621 (1994).

${ }^{15}$ V. L. Sliwko, P. Mohn, K. Schwarz, and P. Blaha, J. Phys.: Con- 
dens. Matter 8, 799 (1996).

${ }^{16}$ P. J. Craievich, J. M. Sanchez, R. E. Watson, and M. Weinert, Phys. Rev. B 55, 787 (1997); Phys. Rev. Lett. 72, 3076 (1994).

${ }^{17}$ M. C. Payne, M. P. Teter, D. C. Allen, T. A. Arias, and J. D. Joannopoulos, Rev. Mod. Phys. 64, 1045 (1992).

${ }^{18}$ D. J. Bottomley and P. Fons, J. Cryst. Growth 160, 406 (1996).

${ }^{19}$ J. Hornstra and W. J. Bartels, J. Cryst. Growth 44, 513 (1978).

${ }^{20}$ K. Yang, T. Anan, and L. J. Schowalter, Appl. Phys. Lett. 65, 2789 (1994).

${ }^{21}$ P. M. Marcus and F. Jona, Phys. Rev. B 51, 5263 (1995).

${ }^{22}$ C. P. Flynn, Phys. Rev. Lett. 57, 599 (1986).

${ }^{23}$ D. M. Wood and A. Zunger, Phys. Rev. Lett. 61, 1501 (1988); Phys. Rev. B 38, 12756 (1988); 40, 4062 (1989).

${ }^{24}$ D. B. Laks, L. G. Ferreira, S. Froyen, and A. Zunger, Phys. Rev. B 46, 12587 (1992).

${ }^{25}$ C. Stampfl and M. Scheffler, Surf. Rev. Lett. 2, 317 (1995).

${ }^{26}$ R. Diehl and R. McGrath, Surf. Sci. Rep. 23, 43 (1996).

${ }^{27}$ R. D. Diehl and R. McGrath, J. Phys.: Condens. Matter 9, 951 (1997).

${ }^{28}$ G. Benedek, J. Ellis, A. Reichmuch, P. Ruggerone, H. Schief, and J. P. Toenneis, Phys. Rev. Lett. 69, 2951 (1992).

${ }^{29}$ E. Hulpke, J. Lower, and A. Reichmuch, Phys. Rev. B 53, 13901
(1996).

${ }^{30}$ M. Bockstedte, A. Kley, J. Neugebauer, and M. Scheffler, Comput. Phys. Commun. 107, 187 (1997).

${ }^{31}$ M. Fuchs, M. Scheffler, Comput. Phys. Commun. 116, 1 (1999).

${ }^{32}$ D. R. Hamann, Phys. Rev. B 40, 2980 (1989).

${ }^{33}$ J. Perdew and A. Zunger, Phys. Rev. B 23, 5048 (1981).

${ }^{34}$ M. G. Gillan, J. Phys.: Condens. Matter 1, 689 (1989).

${ }^{35}$ V. L. Sliwko, P. Mohn, K. Schwarz, and P. Blaha, J. Phys.: Condens. Matter 8, 799 (1996).

${ }^{36}$ G. Simmons and H. Wang, Single Crystal Elastic Constants and Calculated Aggregated Properties: A Handbook, 2nd ed. (MIT Press, Cambridge, MA, 1971).

${ }^{37}$ C. Kittle, Introduction to Solid State Physics, 7th ed. (Wiley, New York, 1996).

${ }^{38}$ M. Fuchs, M. Bockstedte, E. Pehlke, and M. Scheffler, Phys. Rev. B 57, 2134 (1998).

${ }^{39}$ F. M. Wang and R. Ingalls, Phys. Rev. B 57, 5647 (1998).

${ }^{40}$ H. L. Skriver, Phys. Rev. B 31, 1909 (1985).

${ }^{41}$ E. G. Moroni, G. Grimvall, and T. Jarlborg, Phys. Rev. Lett. 76, 2758 (1996).

${ }^{42}$ A. V. D. Walle, G. Ceder, and U. V. Waghmare, Phys. Rev. Lett. 80, 4911 (1998). 